\title{
On Effects of Cloud Computing on Big Data Processing for E-commerce Development
}

\author{
Renhuai Yang ${ }^{1}$, Chuanping Lang ${ }^{1}$ \\ ${ }^{1}$ Computer Engineering Department, Sichuan Vocational and Technical College of Communications, \\ Chengdu, 611130, China
}

Keywords: Cloud computing. E-commerce. Data processing

\begin{abstract}
At present, China's internet technology has gained obvious progress. The development of electronic information technology boosts the development of China's e-commerce to a large extent. Cloud computing is an internet-based calculation mode. Previous IT cannot satisfy requirements of e-commerce data management. Under current cloud computing environment, e-commerce develops better and better. Cloud computing technology contributes to improving e-commerce service efficiency and meanwhile reducing development cost. It owns higher safety and reliability. This paper mainly analyzes specific application of cloud computing in e-commerce development and discusses how it processes data.
\end{abstract}

\section{Introduction}

In current stage, China's e-commerce is in rapid development period. The number of e-commerce users presents the rising trend. Previous electronic data processing structure cannot adapt e-commerce development requirements. In current e-commerce, more data need processing. Besides, the processing process is very complex. Cloud computing technology can provide more data management and owns strong compatibility and better expansibility.

\section{Overview of cloud computing}

After 2007, a new business mode appeared, called “cloud computing”. It is a new internet mode. All computing tasks can be allocated to corresponding computer resources. Besides, storage space and other software services are provided for users on the basis of user needs.

After cloud computing is realized, network services can be conducted via mobile phone or the laptop, which brings convenience for users. It can even accomplish supercomputing. All devices which can be connected to internet can serve as a super computer. Cloud computing has three main features: low cost, high reliability and high expansibility. Currently, the realization of cloud computing technology mainly depends on available resources. It can operate on PC to greatly reduce data processing cost. Since many virtualization technologies are applied in it, many untrusted nodes form. So, designers consider this during the design and finally enhance expansibility and security of cloud computing system.

Cloud computing can provide data storage center for users. Data storage owns very high reliability and avoids virus invasion and loss of data. Besides, loss of data technology does not put forward high requirements for client equipment. So, users can use it very conveniently. Even if the devices applied are different, data sharing can be achieved through cloud computing.

Cloud computing service mode mainly includes three types: platform as a service (PaaS), software as a service (SaaS) and infrastructure as a service (IaaS). Platform as a service means to regard software research and development platform as a service and deliver information to users through SaaS mode. PaaS is a type of SaaS applications. SaaS means software is provided on the basis of internet technology and users do not need purchase the software. It can apply for renting Web software so as to make sure enterprise operation activities can operate normally. IaaS means users can regard the computer as a carrier to gain corresponding services. The services mainly include computing and data storage etc. 


\section{Composition of e-commerce data}

\section{E-commerce module of Taobao}

Take Taobao for example. The structure of Taobao basically includes shopping assistant, information module, payment module, community, user management module, order module, product module and service module. All modules have their own data in need of storage. Seeing from product module, it can be classified into two parts: product management and display. In Taobao, product display is very meticulous. Product management mainly aims at sellers. To attract consumers, most sellers will decorate online shops and manage products. Shopping assistant provides much convenience for consumers, mainly involving shopping through seeing pictures, module search and shopping trolley etc. User management module mainly includes my Taobao and user management. Information module mainly introduces products in detail and provides shopping guide information for consumers. Service module is convenient for refund or complaint handling. Order data in order module belong to core data. Taobao does not own independent logistics. It must deliver order data to logistics to distribute the products. In payment module, users can bind Alipay and Taobao account number to complete payment.

\section{E-commerce module of Jingdong Mall}

Jingdong Mall and Taobao have many common points in terms of user management module, basically including userinfo management, user integral, user data and password change etc. Transaction management mainly manages users' transaction record and order center and evaluates transactions. Service module can realize user complaint and refund management. Community module is mainly a subject forum. Buyers can communicate via posting a message according to product classification. Payment module is mainly realized through mail remittance and Alipay. Order management is convenient for users evaluating, inquiring and modifying the products bought. It is known through analysis that even if e-commerce has differences in the form, there are many common points in the structure.

\section{Effects of cloud computing technology in e-commerce data processing}

The establishment of logic model of e-commerce data means to sort and classify the data related to e-commerce and manage the data through network information organization. Managers can divide data types according to this model. Network information organization mode mainly includes hypermedia, free text, document and homepage. Hypermedia mode means multimedia technology combines with hyper text. Hyper text is applied to reflect multimedia information through integration of information resources. Free text mode refers to unstructured text of organization and management. It puts particular emphasis on database management. Document mode refers to unstructured information of organization and management. Homepage mode refers to comprehensive introduction to an individual or an organization. Online files can form through common organization and integration of these resources. Cloud computing technology has important significance in e-commerce data processing. Its effects are mainly reflected in the following aspects:

\section{Improvement of data processing efficiency}

Cloud computing has large differences with traditional technology. It can realize dynamic resource allocation and release virtual resources and physical resources according to consumer demand. If users have new demand to add, they can add resources and allocate them. If these resources are useless for consumers for the time being, it is necessary to release them. Much space can be saved through releasing resources.

It is because cloud computing can realize dynamic resource allocation that e-commerce enterprises can utilize it to expand application operations. Once visiting traffic is too large, the application system will expand server traffic for the convenience for user access. If users visit little, the system can recover the server to save resources. Generally, e-commerce will launch business promotions activities in a specific time bucket. The types of products for promotion are many and the price is low. At this moment, user page view will very large. Promotion visit is very sudden. If 
internet technology does not prepare sufficiently, system breakdown may occur when a large number of users visit at the same time. After cloud computing technology is applied, such phenomenon can effectively avoid. It owns dynamic transformation property and ca timely provide data traffic.

\section{Rapid information retrieval}

In e-commerce, there are many types of products and competitions in e-commerce industry become increasingly fierce. To dominate in competitions, powerful information retrieval capacity is required for timely retrieving information and classifying products. In this stage, China fails to deeply study database network system. It is still in gradual development period. Dart Grid and My Grid are related to such study. After cloud computing is applied to process a large number of commercial data, it is beneficial to proving users with powerful information retrieval. In this process, user demand and individual differences must be considered to guarantee information reliability and accuracy. Besides, information push function can operate simultaneously to push information some hot products, inform users of new retrieval service and provide more convenience for users. Much work takes specific field as the object and establishes corresponding information service system in static network environment. Since previous data processing technology has many uncertainties, it is required to update processing technology. Cloud computing processing technology contributes to realizing integration of dynamic data, establishing an effective network system and ensuring scientific management of database resources. In the design process, resources gained by users have very high accuracy and the query efficiency can also improve. This is beneficial to managers for scientific management of data resources.

\section{Improvement of data processing efficiency}

In e-commerce data, there are certainly many data in need of analysis. If data analysis efficiency boots, it contributes to e-commerce enterprises to occupy the dominant position in competitions. The value of big data is reflected in taking full advantage of relevant data after data analysis. Through cloud computing, data analysis, processing, storage and collection can be realized. Thus, data analysis and processing ability of e-commerce enterprises can become stronger. E-commerce enterprises can dig more accurate data and deeply analyze data. The operation mode is as follows: based on OGSA-DAI, package all resources in the database, make them become Grid service, register a data warehouse, construct a service framework and apply Shunsaku XML Manager technology to realize data resource management in the resource management warehouse.

Its composition is relatively complex. Firstly, it is required to manage and adjust nodes. In every node, there is prime information corresponding to resources. This greatly influenced nodes. Their respective routing tables will continue to keep. When the system operates, with node quit and join, P2P correctness and integrity are maintained. Resource discovery conducts according to specific information of global schema. Meanwhile, in accordance with semantics of unit information, domain matching and mode matching are achieved. In the process of discovering resources, user need should be considered. Query and processing mainly aim at domain ontology. After request semantics is expanded, query and processing decompose semantics into sub-mode information and inquire resource service information again. At this moment, query execution plan will generate. Data integration is mainly based on subquery technology. During data transmission, the minimum data size will realize integration to optimize field data integration. Replica management utilizes multi-node and multi-backup thought to back up corresponding commercial data and to ensure integrity and effectiveness of service data resource. It contributes to improving network service query efficiency. Executive dispatching carries out global query of subquery in the process of executing dispatching query.

\section{Strong elastic processing capacity}

E-commerce system owns strong elastic processing capacity, which contributes to improving work efficiency of e-commerce enterprises. Once user page view is too much, the system is able to timely deal with it to satisfy user need. Meanwhile, the service can be expended to realize increase in data storage devices.

Cloud storage platform owns rich resources with large storage capacity. It can handle TB-level and PB-level data. Enterprises do not need to install hardware. It can reduce enterprise cost and 
deploy application systems in a short time. It can achieve high limit management of resources and optimization of enterprise resources so as to give full play to its effects.

\section{Realization of data resource sharing via cloud computing technology}

Cloud computing technology can integrate computing resources in different regions via virtualization technology and realize resource sharing. Cloud computing technology provides an application platform for e-commerce enterprises. It can also share multiple product information resources on the basis of the needs of e-commerce enterprises. Cloud computing is a new technology, possesses very high security and reliability, provides convenient services for users and greatly improve work efficiency of e-commerce enterprises.

\section{Conclusions}

The emergence of cloud computing makes e-commerce gain large development space. It improves work efficiency of e-commerce enterprises enormously, reduces enterprise management difficulties and promotes e-commerce development to a large extent. This paper firstly introduces cloud computing in detail, then analyzes the composition of e-commerce data and finally discusses specific effects of cloud computing in e-commerce. It is found through researches that cloud computing is of great significance for e-commerce development.

\section{References}

[1] Zhang Wei, On copyright protection of cloud computing in e-commerce environment [J]. Sci-Tech Information Development \& Economy, 2012 922): 102-104

[2] Jiang Guoyin, Wang Youtian, Ma Feicheng et al., Study on e-commerce solutions based on cloud computing [J]. Mathematics in Practice and Theory, 2013 (08): 151-159

[3] Liu Ling, Jiang Chunyan, Sha Feng, On construciotn of uniform e-government information platform in cloud computing environment [J]. Journal of LuoYang Institute of Science and Technology (Social Science Edition), 2013 (01): 54-57+64

[4] Kang Li, Research of security problems and countermeasures of e-commerce in "cloud computing” environment [J]. Management \& Technology of SME (first), 2013 (02): 283-284

[5] Xing Qiudan, Pu Jianhua, Yan Kang, Analysis of cloud computing solution of B2C e-commerce [J]. Science-Technology and Management, 2011 (05): 48-51

[6] Wang Yijie, Sun Weidong, Pei Xiaoqiang et al., On key technology of distributed storage in cloud computing environment [J]. Journal of Software, 2012 (04): 962-986

[7] Yang Yuhuan, Yang Junqi, Optimization of value chain of B2C e-commerce enterprises based on cloud computing [J]. Enterprise Economy, 2012 (04): 117-120

[8] Yu Yanling, Fang Wenchao, Study on e-commerce development trend based on cloud computing [J]. Financial Economy, 2012 (14): 66-68

[9] Li Keran, Study on e-commerce data management mode based on cloud computing [D]. Xidian University, 2011

[10] Diao Su, Xu Liqun, Lu Tingjie, Study on e-commerce business mode based on social computing environment $[\mathrm{J}]$. Journal of Beijing University of Posts and Telecommunications (social sciences), 2010 (02): 28-33 